\title{
Nanoscale Chemical Imaging of Coking Mechanisms in a Zeolite ZSM-5 Crystal by Atom Probe Tomography
}

Jonathan D. Poplawsky, ${ }^{1}$ Joel E. Schmidt, ${ }^{2}$ Baishakhi Mazumder, ${ }^{1}$ Wei Guo, ${ }^{1}$ Özgün Attila, ${ }^{2}$ Donglong $\mathrm{Fu}^{2}$ D. A. Matthijs de Winter, ${ }^{3}$ Florian Meirer, ${ }^{2}$ Simon R. Bare, ${ }^{4}$ and Bert M. Weckhuysen ${ }^{2}$

1. Center for Nanophase Materials Sciences, Oak Ridge National Laboratory, Oak Ridge, TN, USA.

2. Debye Institute for Nanomaterials Science, Utrecht University, Utrecht, The Netherlands.

3. Structural Geology \& EM, Utrecht University, Utrecht, The Netherlands.

4. SSRL, SLAC National Accelerator Laboratory, Menlo Park, CA,USA.

Zeolites are crystalline microporous materials that are used in large quantities as solid catalysts in oil refining and automotive emission treatments. Due to their widespread use there is much effort to improve their catalytic performance, including activity, selectivity and stability. Coking is known to be a main mechanism for the degradation of zeolite ZSM-5 during reactions with carbon-containing substances, such as methanol. Understanding the nanoscale chemical distribution, such as the location of coke species leading to catalyst deactivation, within zeolites has been a major challenge due to the difficulty in detection of carbon species and electron beam sensitivity when using transmission electron microscopy based techniques. Atom probe tomography (APT) has recently been demonstrated as a useful characterization technique to understand the nanoscale elemental distribution within zeolite materials, allowing for the direct identification of chemical segregation during the processing and reaction of zeolite catalysts. [1-3]

In this work, zeolite ZSM-5 crystals, after reaction for $90 \mathrm{~min}$ at $623 \mathrm{~K}$, in the industrially important methanol-to- hydrocarbons (MTH) reaction have been studied via APT. ${ }^{13} \mathrm{C}$ labeled methanol was used in order to distinguish the reaction carbon species from carbon contamination form specimen preparation or exposure to the environment. ${ }^{13} \mathrm{C}$ labeling was also used to distinguish molecular $\mathrm{C}$ species within the mass spectrum by comparing mass spectra between zeolites rich in ${ }^{13} \mathrm{C}$ versus those rich in ${ }^{12} \mathrm{C}$. After proper identification of the carbon species within the mass spectrum, the coke species were found to form agglomerates tens of nanometers in size as well as small clusters with 30-60 C atoms. Despite a homogeneous distribution of $\mathrm{Al}$ in each sample, the 30-60 atom coke clusters correlate to nanoscale increases in the catalytically active Al sites, known as Brønsted acid sites.

Reacted zeolite ZSM-5 crystals contain Si, Al, O, and hydrocarbons (coke). Properly identifying the hydrocarbons within the mass spectrum is challenging due to the molecular complexity of the evaporated species. To best identify the carbon species, three specimens with different $\mathrm{C}$ compositions and ${ }^{13} \mathrm{C}$ to ${ }^{12} \mathrm{C}$ ratio were run in the atom probe, including a calcined specimen without $\mathrm{C}$, a steamed and reacted specimen with the natural occurring ${ }^{12} \mathrm{C} /{ }^{13} \mathrm{C}$ ratio, and a calcined and reacted specimen enriched in ${ }^{13} \mathrm{C}$. The resulting mass spectra showing the 12 and $13 \mathrm{Da} \mathrm{C}^{+}, 28 \mathrm{Da}$, and $29 \mathrm{Da}$ peaks are displayed in Figure 1 (a), (b), and (c), respectively. Locations of $\mathrm{Si}, \mathrm{SiH},{ }^{13} \mathrm{CO},{ }^{13} \mathrm{CO}$, and ${ }^{13} \mathrm{COH}$ peaks are marked in Figure 1 (b) and (c), which show the identity of the 28 and 29 Da peaks for each specimen. The 28 and 29 Da peak for the sample without $\mathrm{C}$ has a mass that corresponds to $\mathrm{Si}^{+}$, but a slight shift in the 28 and 29 Da peak is observed for the sample containing a natural isotopic ratio of $\mathrm{C}$, which could easily be misidentified as $\mathrm{Si}^{+}$without such a detailed examination. Further examination of the 29/28 $\mathrm{Da}$ and $13 / 12 \mathrm{Da}$ ratios of the ${ }^{13} \mathrm{C}$ rich sample and the alignment of the 28 and $29 \mathrm{Da}$ peaks to ${ }^{12} \mathrm{CO}^{+}$and 
${ }^{13} \mathrm{CO}^{+}$clearly demonstrate that the peak shift is due to the existence of $\mathrm{CO}$ species in the mass spectrum, and indicates that $\mathrm{CO}$ is the main element contributing to the 28 and $29 \mathrm{Da}$ peak for the $\mathrm{C}$ reacted samples. The $\mathrm{CO}$ peaks also contribute to over $75 \%$ of the total $\mathrm{C}$ species in the mass spectrum for $\mathrm{C}$ reacted zeolite ZSM-5 crystals, and therefore, is pertinent to correctly identify the C distribution.

After reaction with ${ }^{13} \mathrm{C}$ labeled methanol to distinguish coke species from contamination, four APT needles were prepared from different depths within a ZSM-5 crystal and analyzed to reveal a corresponding gradient in coke and $\mathrm{Al}$ (catalytically active site) at the $\mu \mathrm{m}$ scale. Clear heterogeneous distributions of the coke content are also realized on the tens of nanometer scale, which can be identified by a $3 \%{ }^{13} \mathrm{C}$ isosurface displayed in Figure $1(\mathrm{~d})$. There also exist ${ }^{13} \mathrm{C}$ clusters within the ${ }^{13} \mathrm{C}$ rich regions, which were first identified by statistically significant deviations in the nearest neighbor distribution analysis. Clusters were extracted from the data using the friends-of-friends cluster analysis with $\mathrm{d}_{\max }$ selected using a double Gaussian fit to the nearest neighbor distribution and $\mathrm{N}_{\min }$ selected using the cluster size distribution algorithm. [4] Extraction of the clusters revealed ${ }^{13} \mathrm{C}$ clusters of the size of typical coke species aggregating in nanoscale regions near catalytically active Al Brønsted acid sites, which could lead to catalyst deactivation. These likely first coke precursors are shown to form throughout the material, which identifies the importance of engineering the Al site density throughout the zeolite ZSM-5 catalyst to suppress deactivation of the material during reaction [5].

\section{References:}

[1] D.E. Perea et al., Nat. Commun,, 6, (2015), p. 7589.

[2] J.E. Schmidt et al., Angew. Chem. Int. Ed, , 128, (2016), p. 11339.

[3] A. Devaraj et al., Sci. Rep, , 6, (2016), p. 37586.

[4] D. Vaumousse, A. Cerezo, P.J. Warren, Ultramicroscopy, 95, (2003), p. 215.

[5] Research was conducted at ORNL's Center for Nanophase Materials Sciences (CNMS), which is a U.S. DOE Office of Science User Facility operated by UT-Battelle, LLC under Contract No. DEAC05-00OR22725.
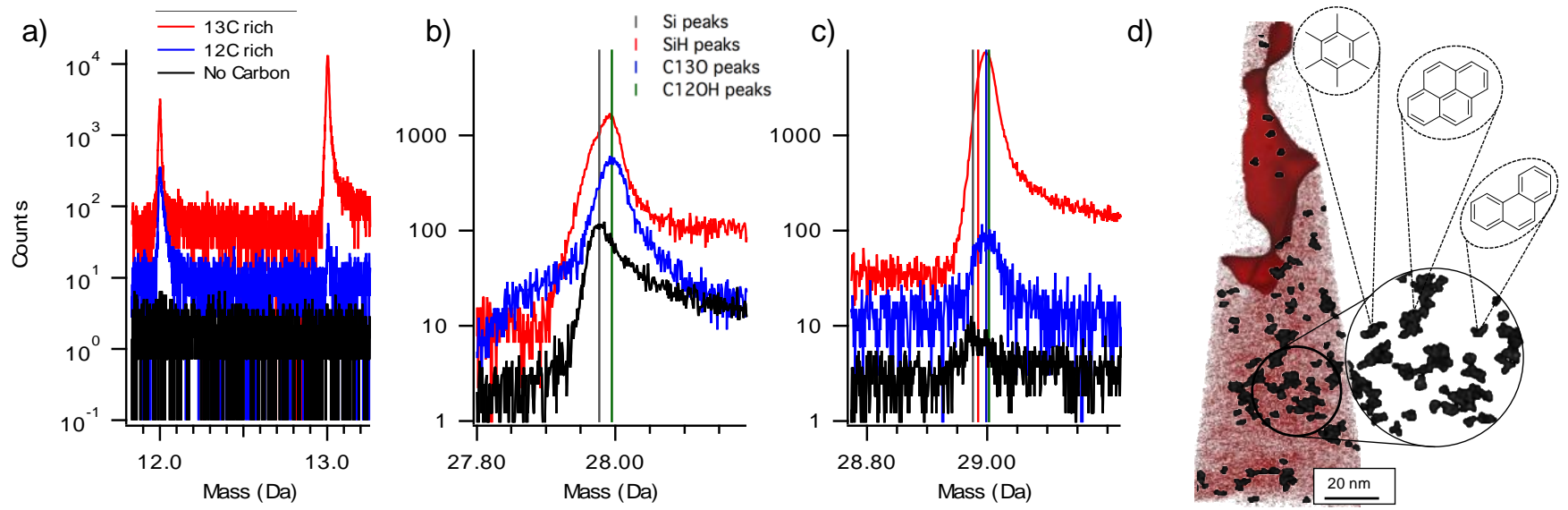

Figure 1. Mass spectra of a zeolite ZSM-5 crystal rich in ${ }^{13} \mathrm{C}$, rich in ${ }^{12} \mathrm{C}$, and without $\mathrm{C}$ centered at (a) ${ }^{13} \mathrm{C}^{+}$and ${ }^{12} \mathrm{C}^{+}$, (b) $28 \mathrm{Da}$, and (c) $29 \mathrm{Da}$ to demonstrate peak shift differences between the three samples, and to identify the peaks corresponding to 28 and 29 Da for each sample. (d) ${ }^{13} \mathrm{C}$ atom map with a 3 at. $\%{ }^{13} \mathrm{C}$ isosurface. Clustering ${ }^{13} \mathrm{C}$ atoms are indicated as black spheres with possible coke species that are consistent with the median ${ }^{13} \mathrm{C}$ cluster size. (d) is also shown in reference [2]. 